reassessment. All children were seen seven days later and stratified according to absence or presence of proteinuria, as a marker for renal involvement, to standard follow up (four in the year) and more frequent visits (seven in the year) if proteinuria present. If proteinuria developed in the standard group, they moved to more frequent follow up. Urinalysis was undertaken at each follow up visit and criteria for consultant review were clearly defined.

(1) Henoch Schonlein Purpura - A 5-Year Review and Proposed Pathway. Louise Watson, et al

Strategy for change During the development stage senior nursing and medical staff were consulted about the practicalities and possibilities of developing a similar pathway and a guideline was produced with department ratification. Only limited information about the pathway was disseminated to junior staff before its introduction.

Measurement of improvement Sixteen months on, an audit and service evaluation was undertaken with feedback from patients, their families and staff.

Effects of changes Of 22 children followed up none developed renal sequelae at any time.

Almost half did not use consultant clinic appointments for follow up with seven completing the pathway appropriately, two having extra nurse follow up and one who had nurse follow up at different intervals to those recommended.

Ten children had consultant follow up, which was not clinically indicated.

Staff feedback was positive. Parents expressed the benefit of more flexible and quicker appointments for monitoring.

Although incomplete concordance with guideline, the process of follow up appears acceptable and manageable to patients, parents and staff. There were clinic slots released and the department generates income for nursing clinic appointments at each review.

Lessons learnt The main lesson learnt was the difficulty in engaging a wide group of people in a change of practice once implemented. This was particularly so when junior staff starting the children on the pathway in the acute phase change frequently. Different ways of working take time to be embedded. To try and mitigate this in future, wider team education is vital at implementation to improve awareness of the change and improve its use in practice.

Message for others To effect change, involving all relevant parties is essential from the start. It is important to educate and inform the wider team when a change of practice is implemented and to have an open dialogue within the team to monitor effectiveness.

\section{G534(P) IMPROVING PAEDIATRIC CLINICAL OUTCOME INDICATORS BY A COLLABORATIVE RETRAINING OF CHILD HEALTH PROFESSIONALS}

${ }^{1} \mathrm{KE}$ Knox, ${ }^{2} \mathrm{P}$ Lutalo, ${ }^{3} \mathrm{~T}$ Ntabayagirwa, ${ }^{4} \mathrm{~A}$ Conayisarye, ${ }^{5} \mathrm{AJ}$ McArdle, ${ }^{6} \mathrm{D}$ Birahinduka. ${ }^{1}$ Paediatrics, North Middlesex University Hospital, London, UK' ${ }^{2}$ Respiratory Medicine, Great Ormond Street Hospital, London, UK; ${ }^{3}$ Paediatrics, Centre Hospitalo-Universitaire de Kamenge (CHUK), Bujumbura, Burundi; ${ }^{4}$ Paediatrics, Hopital Prince Regent Charles (HPRC), Bujumbura, Burundi; ${ }^{5}$ Paediatrics, London North West Healthcare NHS Trust, London, UK; ${ }^{6}$ Paediatrics, University College London Hospital, London, UK

10.1136/archdischild-2015-308599.485

Context PICHEAM (Programme for Improvement of Child Health Hospital Management) was founded by a group of UK healthcare professionals with the objective of reducing childhood hospital mortality from treatable conditions in low-income countries. The project commenced in Burundi in collaboration with staff at two large teaching hospitals.

Problem Healthcare provision in Burundi is under-resourced, with WHO estimates of only 0.028 doctors and 0.19 nurses per 1000 population, and an under-five mortality rate of 83 per 1,000. PICHEAM identified that the two collaborating hospitals did not have local guidelines to standardise emergency management, resulting in significant variation in patient treatment and poor outcomes.

Assessment of problem and analysis of its causes Hospital admission data was analysed at the start of the project focusing on admission diagnoses, treatment plans, hospital stay and mortality. This showed a mortality rate of $6.7 \%$, with over $50 \%$ of children dying on the first day of admission. PICHEAM planned to standardise practice via development of local guidelines and to focus on improving emergency management as a priority.

Intervention Best practice guidelines based on WHO recommendations were drawn up in collaboration with local staff, including protocols for managing emergencies. A simulation-based training package was developed to reinforce teaching of these protocols and develop team working skills, with local staff trained as facilitators thereby establishing a sustainable model.

Study design Morbidity and mortality data are regularly collected by local clinicians using routine hospital data sources.

Strategy for change PICHEAM aims to improve child health outcomes in participating hospitals over a three year period. This involves an initial period of working in partnership to observe practice, collect data, develop guidelines and build relationships with local staff. Guidelines were produced in 'handbook' format and disseminated to hospital staff. Results from audit of admission data were presented at local hospital meetings in Burundi and at PICHEAM meetings in London. Admission data is re-audited and participant feedback from the training sessions collected during each visit to measure change and optimise the teaching package.

Measurement of improvement Data was analysed comparing mortality rate, common diagnosis, length of stay by treated conditions and management in each clinical context.

Effects of changes The number of children diagnosed with pneumonia decreased from 2013 to 2014 (7.2\% vs 10.6\%) in parallel with an increase in rates of bronchiolitis $(6.5 \%$ vs $3 \%)$ following teaching on the differences between the two diagnosis in children under 1 year of age. All children diagnosed with malaria received quinine in 2014 as opposed to $98.2 \%$ in 2013; fewer children with malaria received antibiotic therapy in 2014 compared to $2013(11.6 \%$ vs $14.2 \%)$. The number of children with gastroenteritis treated with intravenous fluids increased $(18.1 \%$ vs $15 \%)$ and more were prescribed antibiotics $(18.4 \%$ vs $4 \%)$. The overall mortality rate as a proportion of all admissions did not show significant difference except after 5 days of hospitalisation (survival rate at day 10, 98.5\% in 2013 vs $97.5 \%$ in 2014). Lessons learnt It is encouraging that there was an increase in bronchiolitis diagnoses, and that fewer children diagnosed with malaria were receiving unnecessary antibiotics. Implementing good data collection is an ongoing process however, and consolidation of work done so far will continue to highlight areas for improvement, such as management of gastroenteritis. The project is continually adapting based on feedback received and learning needs identified, and difficulties in accessing nursing and midwifery staff for training will be addressed in future visits.

Message for others PICHEAM is showing that an ambitious goal can been achieved by simple means; implementation and reinforcement of standardised best practice. 